Published in final edited form as:

Arch Biochem Biophys. 2007 January 1; 457(1): 7-15.

\title{
Characterization of an alternative splice variant of human
} nucleoside triphosphate diphosphohydrolase 3 (NTPDase3): A possible modulator of nucleotidase activity and purinergic

\section{signaling}

\author{
Patrick A. Crawford ${ }^{\#, t}$, Keith J. Gaddie ${ }^{\#}$, Thomas M. Smith\|, and Terence L. Kirley\#, ${ }^{*}$ \\ \# Department of Pharmacology and Cell Biophysics, College of Medicine, University of Cincinnati, P.O. Box \\ 670575, Cincinnati, OH 45267-0575
}

|| Department of Cardiovascular and Metabolic Diseases, Wyeth Research, Cambridge, Massachusetts 02140

\begin{abstract}
Nucleoside triphosphate diphosphohydrolase 3 (NTPDase3) is a cell surface, membrane-bound enzyme that hydrolyzes extracellular nucleotides, thereby modulating purinergic signaling. An alternatively spliced variant of NTPDase 3 was obtained and analyzed. This alternatively spliced variant, termed "NTPDase $3 \beta$ ", is produced through the use of an alternative terminal exon (exon 11) in place of the terminal exon (exon 12) in the full-length NTPDase3, now termed "NTPDase3 $\alpha$ ". This results in an expressed protein lacking the $\mathrm{C}$-terminal cytoplasmic sequence, the $\mathrm{C}$-terminal transmembrane helix, and apyrase conserved region 5. The cDNA encoding this truncated splice variant was detected in a human lung library by PCR. Like the full-length NTPDase $3 \alpha$, the alternatively spliced NTPDase $3 \beta$ was expressed in COS cells after transfection, but only the fulllength NTPDase $3 \alpha$ is enzymatically active and properly trafficked to the plasma membrane. However, when the truncated NTPDase $3 \beta$ was co-transfected with full-length NTPDase $3 \alpha$, there was a significant reduction in the amount of NTPDase $3 \alpha$ that was properly processed and trafficked to the plasma membrane as active enzyme, indicating that the truncated form interferes with normal biosynthetic processing of the full-length enzyme. This suggests a role for the NTPDase $3 \beta$ variant in the regulation of NTPDase 3 nucleotidase activity, and therefore the control of purinergic signaling, in those cells and tissues expressing both NTPDase $3 \alpha$ and NTPDase $3 \beta$.
\end{abstract}

\section{Keywords}

nucleoside triphosphate diphosphohydrolase 3 (NTPDase3); alternative splicing; biosynthetic processing; cellular trafficking; expression modulation; CD39L3

\footnotetext{
* To whom correspondence should be addressed: Dr. Terence L. Kirley, Department of Pharmacology and Cell Biophysics, University of Cincinnati College of Medicine, 231 Albert Sabin Way, Cincinnati, OH 45267 Phone: 513-558-2353 Fax: 513-558-9969 E-Mail: terrry.kirley@uc.edu.

7 current address: Department of Chemistry, Augustana College, Rock Island, IL 61201

Publisher's Disclaimer: This is a PDF file of an unedited manuscript that has been accepted for publication. As a service to our customers we are providing this early version of the manuscript. The manuscript will undergo copyediting, typesetting, and review of the resulting proof before it is published in its final citable form. Please note that during the production process errorsmaybe discovered which could affect the content, and all legal disclaimers that apply to the journal pertain.
} 


\section{Introduction}

The nucleoside triphosphate diphosphohydrolases (NTPDases) are a family of enzymes known to hydrolyze a variety of nucleoside 5'-triphosphates and 5'-diphosphates. These enzymes are found in mammals, including humans, and are also found in insects, plants, yeast, invertebrates, and protozoa [1,2]. There are eight known members of this family: NTPDase1-3 and 8 hydrolyze nucleotides extracellularly, NTPDase 4 and 7 hydrolyze intralumenal nucleotides, and NTPDase5 and 6 can hydrolyze nucleotides in both locations [3]. NTPDase1-4, 7, and 8 are always membrane-bound, having two membrane-spanning domains, one each at the $\mathrm{C}$ - and $\mathrm{N}$-termini of the proteins [3]. NTPDase 5 and 6 have a unique topology in that they have just one membrane-spanning domain and, after cleavage of these respective $\mathrm{N}$-terminal signal peptides, they can be released into the extracellular space as soluble enzymes [4-9]. NTPDases that are found at the cell surface modulate signaling mediated by cell-surface purinergic receptors, which are known to control many physiological processes, including blood clotting, pain perception, and smooth muscle contraction $[1,10]$.

The members of the NTPDase family differ considerably in their nucleotide hydrolysis specificities and relative efficiencies of hydrolysis of di- versus tri-phosphate nucleotides. For example, NTPDase 1 (aka ecto-apyrase, CD39) hydrolyzes di- and triphosphates almost equally well (ATPase:ADPase ratio $\approx 1$ ), while NTPDase2 (ecto-ATPase) preferentially hydrolyzes triphosphates (NTPase:NDPase ratios greater than 25:1). NTPDase5 and 6 greatly prefer diphosphates over triphosphates as substrates [4-9]. These differential nucleotide hydrolysis profiles are important for distinguishing the functions of these various NTPDases [10]. Despite these differences in substrate specificity, all family members are characterized by the presence of seven highly conserved domains, called apyrase conserved regions (ACRs [11,12]) and by their dependence upon divalent cations $\left(\mathrm{Mg}^{2+}\right.$ or $\mathrm{Ca}^{2+}$ ) for activity. Human NTPDase3 (aka HB6 [13] or CD39L3 [14]) is a membrane bound NTPDase that is characterized by two transmembrane domains, 7 potential $\mathrm{N}$-glycosylation sites, and 10 extracellular cysteines that form 5 disulfide bonds [15]. NTPDase3, with an ATPase:ADPase ratio of 3-5 [13], is a functionally intermediate enzyme compared to the other two well-characterized cell surface NTPDases, NTPDase1 (ATPase:ADPase ratio $\approx 1$ ) and NTPDase2 (NTPase:NDPase > 25:1).

Alternatively spliced variants have been reported for many enzymes, including some NTPDases. In fact, the originally described clone encoding human NTPDase2 (CD39L1) turned out to be an alternatively spliced form that lacked 23 amino acids of the extracellular domain [16]. More recently, three splice variants of NTPDase2 were reported, with NTPDase $2 \alpha$ being the full-length, active form of NTPDase2, NTPDase $2 \beta$ being the isoform originally reported (CD39L1 [16]), and NTPDase $2 \gamma$ being another truncated NTPDase2 isoform [17]. Only the full-length NTPDase2, NTPDase2 $\alpha$, has ATPase activity and is fully processed and trafficked to the plasma membrane [17]. The two splice variants of NTPDase2 ( $2 \beta$ and $2 \gamma$ ) are due to differential splicing of NTPDase 2 exon 8 . The resulting truncated splice variants are inactive, lack a portion of the extracellular domain near ACR5, are not fully biosynthetically processed, and are retained in the endoplasmic reticulum [17]. A variant of rat NTPDase 2 has been reported to have an extended open reading frame due to the alternative splicing loss of a 193 bp exon, which contained the stop codon $[18,19]$. This alternatively spliced cDNA results in a translated protein product with an additional 50 amino acids on the cytoplasmic C-terminal domain, encoding three potential protein kinase (CK2)

phosphorylation sites not present in the original rat NTPDase 2 clone. An active, alternatively spliced NTPDase4 (aka LALP70), differing in 8 amino acids, has been described and was shown to also be dependent on $\mathrm{Ca}^{2+}$ and $\mathrm{Mg}^{2+}$ for nucleotidase activity, but with a broader nucleotide substrate specificity [20]. Additionally, a truncated splice variant of NTPDase1, obtained from a human placental cDNA library, has been reported but not yet fully characterized [21]. 
Here we report the characterization of an alternative splice variant of NTPDase3. The cDNA of this splice variant, termed NTPDase $3 \beta$, codes for a truncated protein that contains all of the cysteines required for the five extracellular disulfide bonds [15], but is missing the C-terminal 77 amino acids, and therefore is lacking a portion of the extracellular domain containing ACR5, one of the N-glycosylation sites (N454), the C-terminal transmembrane domain, and the short cytoplasmic tail sequence. The cDNA encoding this inactive form of NTPDase 3 was detected in a human lung library by PCR. Expression of this truncated splice variant results in an inactive, incompletely processed protein that suppresses the expression level of co-transfected, full-length, enzymatically active NTPDase $3 \alpha$.

\section{Material and Methods \\ IMAGE Clone encoding NTPDase $\beta \beta$}

A bacterial stock of the NTPDase $3 \beta$ IMAGE clone (an alternatively spliced human NTPDase3, IMAGE ID 5174918; GenBank accession BC029869) in pCMV-SPORT6 vector was obtained from Invitrogen. Plasmid DNA was purified (Qiagen MiniPrep) and sequenced (University of Cincinnati DNA Core Facility), and digested with NotI and KpnI (Fisher Scientific). A 1\% low-melting agarose gel was used to purify the gene-containing plasmid insert after endonuclease digestion. The resulting fragment was ligated using T4 DNA Ligase (Invitrogen) into mammalian expression vector pcDNA3, which had also been digested with the same endonucleases. The resulting plasmid was transformed into XL1-Blue Supercompetent bacterial cells (Stratagene) and plated on carbenecillin containing LB-Agar plates.

\section{Site-Directed Mutagenesis of NTPDase3}

Mutagenesis of wild-type NTPDase $3 \alpha$ in pcDNA3 vector was performed using a QuikChange Site Directed Mutagenesis kit (Stratagene) as previously described [22]. The DNA Core Facility at the University of Cincinnati produced the synthetic oligonucleotide primers needed to engineer an NTPDase 3 mutant lacking the 19 amino acids comprising the C-terminal cytoplasmic tail (termed "A511Stop", sense primer: 5'-

GCATACCTGTGTTCATGAACCAGAAGAAAGAGGC-3' and antisense primer: 5'GCCTCTTTCTTCTGGTTCATGAACACAGGTATGC-3'), and sequenced the resulting cDNA construct to verify the presence of the desired mutation and the absence of any unwanted changes.

\section{Selective PCR of NTPDase3 Isoforms}

Three Life Technologies human SuperScript cDNA libraries (from lung, brain and heart tissues) were screened for NTPDase3 isoforms. The primers used either recognize both fulllength $(\alpha)$ and truncated ( $\beta$ ) NTPDase3; P-1 (5'-CACGGATCCACCCCCATTCAT-3') and P-2 (5'-CCACCCAAATATCCTTCG-3'); or are specific for full-length NTPDase $3 \alpha$, P- $\alpha$ (5'CTTACTCTTCCTCTGGGG-3'); or are specific for truncated NTPDase3 $\beta$, P- $\beta$ (5'-

TCAGAGGCCTAATGCAGGAT-3'). The location and specificity of the primers used are indicated in Figure 2. Both the full-length and truncated isoforms of NTPDase3 were amplified directly from the cDNA library by PCR incorporating the P-1/P- $\beta, \mathrm{P}-2 / \mathrm{P}-\beta$, or $\mathrm{P}-1 / \mathrm{P}-\alpha$ primer pairs using a FastStart Taq DNA Polymerase PCR kit (Roche). 25 cycles of PCR were performed as follows: $95^{\circ} \mathrm{C}$ denaturation for $30 \mathrm{sec}, 55^{\circ} \mathrm{C}$ annealing for $1 \mathrm{~min}, 68^{\circ} \mathrm{C}$ extension for 10 min using a Thermolyne Amplitron I thermocycler. PCR products specific for NTPDase $3 \beta$ were ligated into a TOPO vector (Invitrogen TOPO TA Cloning kit). After purification of the plasmid DNA (Qiagen MiniPrep), the identities of the NTPDase3 $\beta$ PCRamplified fragments were confirmed by sequencing. 


\section{Transient Transfection and Preparation of COS Cell Membranes}

COS-1 cells were grown in Dulbecco's modified Eagle's medium (DMEM) with $10 \%$ bovine serum and antibiotics and antimycotics (Invitrogen) [13]. Cells were transfected with a total of $4 \mu \mathrm{g}$, or $8 \mu \mathrm{g}$ in co-transfection experiments, plasmid DNA per 100mm plate using Lipofectamine and PLUS reagents (Invitrogen) as previously described [13]. Transfection with $4 \mu \mathrm{g}$, or $8 \mu \mathrm{g}$ for co-transfection experiments, of empty pcDNA3 vector was performed as a control. The COS- 1 cells were harvested approximately 48 hours post-transfection. The crude cell membrane preparations were obtained as previously described [13].

\section{Protein Assay}

Protein concentrations were determined using the Bio-Rad protein assay reagent with the modifications of Stoscheck [23]. Bovine serum albumin was used as the standard.

\section{Nucleotidase Assay}

Nucleotidase activities were determined by measuring the amount of inorganic phosphate ( $\mathrm{Pi})$ released from nucleotide substrates (Sigma) at $37^{\circ} \mathrm{C}$ using modifications of the technique of Fiske and Subbarow [24] as previously described [22]. Either $5 \mathrm{mM} \mathrm{MgCl}_{2}$ or $5 \mathrm{mM} \mathrm{CaCl}_{2}$ was used for cation dependent nucleotidase activity. The reactions were initiated by the addition of nucleotide to a final concentration of $2.5 \mathrm{mM}$ in the $0.3 \mathrm{~mL}$ assay solutions. Hydrolysis was allowed to proceed for 30 minutes or 1 hour, depending on the substrate used. The activities were corrected for pcDNA3/COS-1 cell background (COS cells transfected with an empty vector), as well as differences in expression levels as determined by quantitative Western blotting of each sample.

\section{SDS-PAGE and Western Blotting}

Pre-cast 10-well or 15-well 4-15\% gradient minigels were purchased from Bio-Rad and used to resolve aliquots of membrane proteins $(0.5-2 \mu \mathrm{g}$, depending on the sample and experimental purpose). After electrophoresis, the proteins were electrotransferred to Immun-Blot PVDF membranes (Bio-Rad) and processed as previously described [25], using a 1:5000 dilution of polyclonal primary antisera incubated overnight at room temperature, and a 1:2000 dilution of stabilized goat anti-rabbit HRP-conjugated secondary antibody (Pierce) for 1 hour at room temperature. Immunoreactivity was detected by chemiluminescence after using Pierce SuperSignal West Dura Extended Duration Substrate for 5 minutes. Chemiluminescence was recorded and quantified using a FluorChem IS-8800 system (Alpha Innotech). Two rabbit polyclonal primary antisera were used, KLH1 and KLH11. KLH1 antisera was generated against the cytoplasmic C-terminal peptide (amino acid residues 515-529) of human NTPDase3 [25]. KLH11 antisera was generated against an amino acid sequence in the extracellular domain of human NTPDase3 (amino acid residues 311-327) and has been previously described [15]. All expressed NTPDase 3 proteins are reactive with KLH11, while both NTPDase $3 \beta$ and the A511Stop NTPDase3 mutant are non-reactive with KLH1, since they lack the C-terminal cytoplasmic amino acid sequence found in full-length NTPDase3 (NTPDase3 $\alpha$ ) used to generate the KLH1 anti-peptide antisera (see Figure 2).

\section{Deglycosylation}

Deglycosylation was performed using Endo $\mathrm{H}$ (New England Biolabs) according to the manufacturer's instructions, as previously described [15]. Briefly, crude membrane preparations were solubilized with $0.2 \%$ Triton X-100 containing 1 mM EDTA for 30 minutes at room temperature. The solubilized samples were then centrifuged in a Beckman air-driven centrifuge (Airfuge) for 20 minutes at 100,000 x g. The supernatant was combined with 10X Glycoprotein Denaturing Buffer ( $0.5 \%$ SDS and $1 \% \beta$-mercaptoethanol) and boiled for 10 minutes. After cooling to room temperature, each sample was combined with the appropriate 
reaction buffer (NEBuffer G5), aliquoted, and incubated with, or without as a control, Endo $\mathrm{H}$ at $37^{\circ} \mathrm{C}$ for 1 or 3 hours. After incubation, the samples were combined with SDS sample buffer containing $100 \mathrm{mM}$ DTT and analyzed by Western blot.

\section{Results NTPDase $3 \beta$ alternatively spliced isoform}

NTPDase3 $\beta$, a truncated, alternatively spliced, variant of NTPDase3, was discovered by searching the IMAGE [26] clone libraries (IMAGE ID 5174918; GenBank accession BC029869). The complete 1608 bp cDNA coding for NTPDase $3 \beta$ was sequenced and found to be a novel isoform of NTPDase3, with the coding region of NTPDase $3 \beta$ identical to the fulllength NTPDase $3 \alpha$, with two notable exceptions (Figure 1): a single A--G point mutation changing Ile24, at the beginning of the N-terminal transmembrane domain in NTPDase $3 \alpha$, to $\mathrm{Val}$ in NTPDase $3 \beta$ (this I $24 \mathrm{~V}$ point mutation is a known single nucleotide polymorphism in NTPDase3, according to the HapMap database at http://www.hapmap.org/) and, more importantly, a premature truncation of NTPDase $3 \beta$ after Glu452, which is a Val in NTPDase $3 \alpha$. Additionally, the cDNA encoding the NTPDase $3 \beta$ isoform has a shorter 5'untranslated region. The resulting translated product of NTPDase $3 \beta$ cDNA is a truncated protein maintaining the 10 cysteines forming 5 disulfides [15], however it is lacking one of the apyrase conserved regions (ACR5), a potential N-glycosylation site (Asn454), the C-terminal transmembrane helix, and the short cytoplasmic C-terminal peptide.

This truncation is generated using an alternative exon in the genomic DNA of NTPDase 3 (Figure 2). Thus, both isoforms of NTPDase 3 consist of 11 exons, the first 10 of which are the same. The alternative exons, exons 11 and 12 , are the terminal exons of NTPDase $3 \beta$ and NTPDase $3 \alpha$, respectively. The difference in the 5 '-untranslated regions results from NTPDase $3 \beta$ having only nucleotides 59 through 68 of exon 1 , presumably due to the use of an alternative promoter.

\section{Isolation of NTPDase $3 \beta$ from a cDNA Library}

To demonstrate that this truncated isoform of NTPDase 3 was not an artifact of the IMAGE clone generation process, PCR amplification was utilized to selectively isolate fragments of the two isoforms of NTPDase3 from a human lung cDNA library, as shown in Figure 3. Human brain and heart cDNA libraries were also screened for these two isoforms using the same PCR primers, but since preliminary PCR results were negative, the lung NTPDase $3 \beta$ isoform was characterized, rather than continuing to look for, or attempting to optimize conditions to detect, the beta isoform in other tissue libraries. The PCR products of the expected size were observed for the NTPDase $3 \beta$ isoform using the lung library, and these products were cloned and sequenced for comparison to the sequences of NTPDase $3 \alpha[13]$ and NTPDase3 $\beta$ (Figure 1). The sequences of the two PCR product fragments were found to be identical to that of NTPDase3 $\beta$ (data not shown).

\section{Expression and Enzymatic Activities of NTPDase3 Isoforms}

The isoforms of NTPDase 3 were expressed in COS-1 cells. Western-blot analysis, utilizing an antibody against an internal peptide sequence (KLH11, see Figure 2), revealed that the membrane fraction of the COS cell preparations contained the NTPDase $3 \beta$ protein.

NTPDase $3 \beta$ was not detectable in the culture media, indicating it is not released as a secreted, soluble form (data not shown). Additionally, Western blot analysis revealed a difference in the molecular masses of the two isoforms of NTPDase 3 as predicted from their respective amino acid sequences (see Figure 4, the non-glycosylated theoretical molecular mass of the full-length NTPDase $3 \alpha$ isoform is $59,190 \mathrm{Da}$, compared to $50,748 \mathrm{Da}$ for the non-glycosylated NTPDase $3 \beta$ isoform). 
Hydrolysis of ATP and ADP was analyzed in the crude membrane preparations of COS- 1 cells that had been transiently transfected with NTPDase $3 \alpha$ or NTPDase $3 \beta$, as well as in the cell culture media, to determine if the truncated form of NTPDase 3 was being released as a soluble form after cleavage of the single transmembrane helix, similar to what has been observed for NTPDase 5 and 6 . Nucleotide hydrolysis by NTPDase $3 \alpha$ was, as expected, detected in the membrane preparations $[13,15,22]$. However, there was no measurable activity detected for NTPDase $3 \beta$ in the membrane preparations, or in any of the soluble fractions (data not shown). COS cell membrane fractions expressing NTPDase $3 \beta$ protein were further tested for activity using alternative nucleotide substrates (AMP, GDP, UDP, UTP, IDP, CDP, and CTP). However, no detectable hydrolysis was observed using any of the nucleotide substrates.

\section{Co-Transfection of NTPDase $3 \alpha$ and NTPDase $3 \beta$}

COS- 1 cells were transfected with NTPDase $3 \alpha$, NTPDase3 $\beta$, the A511Stop-NTPDase3 mutant, or a combination of the three cDNAs, as indicated in Table 1. Crude membrane preparations were then prepared as above and the resulting samples were characterized for expression of NTPDase3 protein by Western blots (Figure 4) as well as for nucleotidase activities (Table 1 and Figure 5). The A511Stop mutant is a fully active NTPDase $3 \alpha$ mutant lacking only the cytoplasmic C-terminal sequence (i.e. amino acids 511-529) and therefore not reactive to the C-terminal antibody KLH1, but still reactive to the internal antibody KLH11. These properties allowed the A511Stop mutant to be used as a control in the co-transfection experiments.

ATPase and ADPase activity assays showed decreases in specific activity for the NTPDase $3 \beta$ containing co-transfections that were substantially greater than can be explained by NTPDase $3 \beta$ simply being an inactive isoform and having no effect on NTPDase $3 \alpha$ expression and activity (Table 1). These activities are approximately $40 \%$ of the nucleotidase activities measured for COS cells transfected with NTPDase $3 \alpha$ alone (Table 1, compare the " $8,0,0$ " row with the " $4,4,0$ " row). Smaller decreases in activity are also seen when NTPDase $3 \alpha$ is co-transfected with NTPDase $3 \beta$ and A511Stop (the " $4,2,2$ " row). Importantly, the relatively small decreases in activity and expression levels seen when co-expressing the active A511Stop mutant with the full-length NTPDase $3 \alpha$ are substantially and statistically significantly different in every case than those observed when co-expressing NTPDase $3 \alpha$ with NTPDase $3 \beta$ (compare the " $4,0,4$ " and " $4,4,0$ " rows in Table 1). The comparison of these two rows is the most important aspect of Table 1 and shows a clear difference between a "control" non-interfering co-transfected DNA (A511Stop in the "4,0,4" row) and a co-transfected DNA that results in inhibition of expression of full-length wild-type enzyme (the NTPDase $3 \beta$ isoform, in the "4,4,0" row). This is a specific effect of the presence of NTPDase $3 \beta$, since the presence of the active, normally processed, artificial truncation mutant, A511Stop, only decreases NTPDase $3 \alpha$ expression level by about 40\% (see Table 1 and lanes 1 and 2 and 6 and 7 of Figure 4). Specifically, the relative protein expression level of NTPDase $3 \alpha$ in the presence of A511Stop ( $60 \pm 14 \%)$ is statistically different than its expression in the presence of the same amount of DNA encoding NTPDase $3 \beta(30 \pm 6 \%)$. The active and properly processed A511Stop was used as a control for non-specific effects that might be caused by any other DNA present during co-transfection, affecting the amount of DNA taken up by the COS cells, or the ability of the COS cells to express the transiently transfected NTPDase $3 \alpha$ cDNA.

Using KLH11, which detects all NTPDase3 proteins used in this study (Figure 4), we are able to distinguish, on the basis of size, between NTPDase $3 \alpha$ and NTPDase $3 \beta$ by Western blot analysis, as well as between A511Stop and NTPDase3 $\beta$, but unable to distinguish between NTPDase $3 \alpha$ and A511Stop (Figure 4). Thus, KLH11 can be used to distinguish between the active forms of NTPDase3 (NTPDase3 $\alpha$ and A511Stop - the upper bands in lanes 6-9 in Figure 4) and the inactive NTPDase $3 \beta$ (the lower bands in lanes 8-10 in Figure 4). Therefore, the data 
plotted in Figure 5 is calculated from the nucleotidase activities reported in Table 1, after correcting for the amount of active and properly processed NTPDase 3 by dividing the specific activities in Table 1 by the relative densities of the upper, active band detected by the KLH11 antibody (as depicted in lanes 6-9 of Figure 4 and quantitated in Table 1). As seen in Figure 5 , the activity per molecule of fully processed NTPDase3 (NTPDase3 $\alpha$ plus A511Stop) is not changed by the presence of either 2 or $4 \mu \mathrm{g}$ of NTPDase $3 \beta$ DNA, suggesting that the coexpression of NTPDase $3 \beta$ does not inactivate NTPDase $3 \alpha$ (or A511Stop) through a direct protein-protein interaction, as might occur via hetero-oligomerization-induced inactivation of NTPDase $3 \alpha$ by NTPDase $3 \beta$ contained in the same oligomer. Thus, both NTPDase $3 \alpha$ and the active A511Stop mutant exhibit the same nucleotidase activity per molecule expressed either in the absence of the presence of co-expressed NTPDase $3 \beta$, as quantified by the upper band reactive with the KLH11 antibody. This results in no significant differences between any of the nucleotidase activities, after correction for expression level (see Figure 5, where none of the values are statistically different from the " $8,0,0$ " row controls). This suggests that the presence of NTPDase $3 \beta$ protein interferes with the bio-processing of NTPDase $3 \alpha$ protein, decreasing the amount of NTPDase $3 \alpha$ that is properly processed and delivered to the cell membrane in an active form, but does not alter the specific activity of the decreased amount of NTPDase $3 \alpha$ that is properly processed.

\section{Biosynthetic processing of NTPDase $3 \alpha$ and NTPDase $3 \beta$ expressed in COS cells}

We previously established that the glycosidase Endo H can be used as a simple tool to analyze the biosynthetic processing of NTPDase3, and that conclusions similar to those reached by performing cell surface biotinylation experiments can be made based on the simpler Endo $\mathrm{H}$ assay [15]. Thus, wild-type NTPDase3, when fully processed and delivered to the cell surface, exhibits a very slight increase in electrophoretic mobility after Endo $\mathrm{H}$ treatment, suggesting that only one, or perhaps two, of the seven N-linked glycosylation sites are not fully processed and therefore removed by Endo $\mathrm{H}$ digestion [15]. This is in contrast to some mutations of NTPDase 3 which are not processed normally and are not delivered to the cell surface (as assessed by cell surface biotinylation experiments published previously [15]). For these improperly processed mutants, Endo H digestion is complete, resulting in a homogenous, narrow protein band on Western blots, which electrophoreses just above the position of the protein core band observed upon complete deglycosylation by peptide $\mathrm{N}$-glycosidase $\mathrm{F}$ (PNGase-F) treatment [15]. When COS cell expressed NTPDase3 $\beta$ is analyzed in this fashion, Endo $\mathrm{H}$ digestion is complete (see Figure 6, lanes 4 and 6), indicating that this alternatively spliced protein is not glycosylated normally in the Golgi complex, but instead most likely retained in the endoplasmic reticulum. This is observed both when NTPDase $3 \beta$ is transfected alone (lanes 5 and 6 in Figure 6) and when it is co-transfected with NTPDase $3 \alpha$ (lanes 3 and 4 in Figure 6).

\section{Discussion}

In this report a splice variant of the NTPDase3 gene is identified and characterized. Sequence analysis of the cDNA indicated the differences between the previously fully characterized, fulllength NTPDase 3 and the new variant is an alternative splice event involving the exon encoding the $\mathrm{C}$-terminus. Expression of the splice variant indicates that the splicing used to generate the full-length human NTPDase 3 is essential for the proper folding, trafficking, and activity of the enzyme. However, the truncated isoform, NTPDase $3 \beta$, maintains all of the cysteines involved in the five disulfide bonds required for the proper folding of NTPDase3 [15], including C422 (C399 in NTPDase2) that was shown to be essential for activity of NTPDase2 and was lacking in both of the NTPDase 2 truncated splice variants [17]. 
Comparison of the sequences of NTPDase $3 \beta$ with the full-length NTPDase $3 \alpha$ shows that the missing portion of the extracellular domain consists of a short $\beta$-sheet connecting to an $\alpha$-helix containing ACR5, according to the 3-D model recently generated for the NTPDases [15]. The missing extracellular domain does not directly involve the phosphate binding motifs located in ACR1 and ACR4. The importance of the membrane spanning regions for NTPDase activities have been demonstrated for NTPDase1 [27-29], NTPDase2 [30], and NTPDase8 [31]. The movement of the transmembrane domains observed for NTPDase1 [29], including the Cterminal transmembrane helix lacking in NTPDase $3 \beta$, have been speculated to be linked to movements in the extracellular lobes which define the active site and are therefore essential for nucleotide hydrolysis [32]. The truncated NTPDase 3 variant described in the current study is also missing the short C-terminal cytoplasmic domain. However, this part of the protein is neither needed for proper trafficking to the cell membrane nor for enzymatic activity of NTPDase3, as demonstrated by the fully active A511Stop NTPDase3 $\alpha$ mutant used in this study.

The physiological significance of the existence of multiple variants for several NTPDases, most of which produce improperly processed and inactive proteins, is unknown. However, the presence of alternative splicing in multiple species and multiple members of the NTPDase family suggests that alternative splicing may be a common mechanism for the regulation of these enzymes. Since NTPDases form active oligomeric structures, the inactive, truncated splice variants might form hetero-oligomeric structures with the full-length isoforms, thereby inactivating the full-length forms, as suggested by Mateo et al. [17]. However, based on data presented in this study, these hetero-oligomers of NTPDase3, if formed, do not inactivate the full-length NTPDase $3 \alpha$ contained in these speculative hetero-oligomers. If this were the case, the activity per molecule of NTPDase $3 \alpha$ would be expected to decrease in co-transfection experiments including the truncated form. This is not the case, since neither of the co-expressed, active NTPDase 3 proteins (neither NTPDase $3 \alpha$ nor the A511Stop mutant) have statistically different nucleotidase specific activities per molecule compared to the NTPDase $3 \alpha$ enzyme expressed alone (see Figure 5). Thus, this putative inactivation by hetero-oligomer formation between a full-length form and a truncated form does not take place for NTPDase 3 and its shortened form.

The conclusion that the NTPDase $3 \beta$ is not delivered to the cell surface where it could form hetero-oligomers with NTPDase $3 \alpha$ is confirmed by the lack of proper glycosylation of the truncated enzyme, as measured by its sensitivity to Endo H digestion (see Figure 6). This situation is in contrast to what has been found for the $\mathrm{P}_{2} \mathrm{X}_{7}$ receptor, which has identical membrane topology to the cell surface NTPDases [33], and for the human serotonin receptor 5-HT3A [34,35], which both form hetero-oligomers containing both full-length and truncated proteins. That this mechanism of inactivation by hetero-oligomerization does not apply to fulllength NTPDase $3 \alpha$ and its truncation variant is also supported by the lack of coimmunoprecipitation of the truncated form with full-length NTPDase $3 \alpha$, using the KLH1 antibody specific for the full-length form (data not shown). Co-immunoprecipitation would be expected if this mechanism was relevant to NTPDase3, and was in fact observed for the purinergic $\mathrm{P}_{2} \mathrm{X}_{7}$ receptor and its truncated form, which seems to regulate the full-length isoform of that receptor by formation of inactive hetero-oligomers containing the truncated form [35]. Instead, in this study, the alternatively spliced, truncated NTPDase 3 is not properly processed and is not delivered to the cell surface, as judged by Endo $\mathrm{H}$ results (Figure 6). More importantly, NTPDase $3 \beta$ appears to be interfering with the processing of the full-length isoform (as judged by the large decrease in expression levels of the NTPDase $3 \alpha$ when cotransfected with NTPDase3 $\beta$ (Table 1 and Figure 4)). That this is not some non-specific effect of the presence of another cDNA co-expressed by the same COS cells is supported by the much smaller, and statistically different effect observed when the fully processed, and active, A511Stop mutant is co-transfected along with NTPDase $3 \alpha$ (see Table 1). It also seems unlikely 
that the effect of the co-transfection of NTPDase $3 \beta$ on NTPDase $3 \alpha$ expression is mediated via transcriptional means. Thus, we speculate that the physiological role for the enzymatically inactive, truncated isoform of NTPDase 3 described in this work might be to decrease the ATPase and ADPase activities at the cell surface due to lessened synthesis and cell-surface delivery of the full-length NTPDase $3 \alpha$, which in turn would lead to extended times of activation of $\mathrm{P}_{2}$ purinergic receptors by extracellular nucleotides, and increased $\mathrm{P}_{2}$ signaling in cells expressing the truncated isoform of NTPDase 3.

\section{Acknowledgements}

This work was supported by NIH grants HL59915 and HL72882 to T.L.K.

\section{References}

1. Plesner L. International Review of Cytology 1995;158:141-214. [PubMed: 7721538]

2. Zimmermann, H.; Beaudoin, AR.; Bollen, M.; Goding, JW.; Guidotti, G.; Kirley, TL.; Robson, SC.; Sano, K. Second International Workshop on Ecto-ATPases and Related Ectonucleotidases. Vanduffel, L., editor. Shaker Publishing BV, Maastricht; The Netherlands, Diepenbeek, Belgium: 1999. p. 1-9.

3. Zimmermann H. Trends in Pharmacological Sciences 1999;20:231-6. [PubMed: 10366865]

4. Hicks-Berger CA, Chadwick BP, Frischauf AM, Kirley TL. Journal of Biological Chemistry 2000;275:34041-34045. [PubMed: 10948193]

5. Mulero JJ, Yeung G, Nelken ST, Bright JM, McGowan DW, Ford JE. Biochemistry 2000;39:1292412928. [PubMed: 11041857]

6. Yeung G, Mulero JJ, McGowan DW, Bajwa SS, Ford JE. Biochemistry 2000;39:12916-12923. [PubMed: 11041856]

7. Braun N, Fengler S, Ebeling C, Servos J, Zimmermann H. Biochemical Journal 2000;351:639-647. [PubMed: 11042118]

8. Ivanenkov VV, Murphy-Piedmonte DM, Kirley TL. Biochemistry 2003;42:11726-11735. [PubMed: 14529283]

9. Murphy-Piedmonte DM, Crawford PA, Kirley TL. Biochimica et Biophysica Acta 2005;1747:251259. [PubMed: 15698960]

10. Kukulski F, Lévesque SA, Lavoie EG, Lecka J, Bigonnesse F, Knowles AF, Robson SC, Kirley TL, Sévigny J. Purinergic Signalling 2005;1:193-204.

11. Handa M, Guidotti G. Biochemical and Biophysical Research Communications 1996;218:916-923. [PubMed: 8579614]

12. Kirley TL, Yang F, Ivanenkov VV. Archives of Biochemistry and Biophysics 2001;395:94-102. [PubMed: 11673870]

13. Smith TM, Kirley TL. Biochimica et Biophysica Acta 1998;1386:65-78. [PubMed: 9675246]

14. Chadwick BP, Frischauf AM. Genomics 1998;50:357-367. [PubMed: 9676430]

15. Ivanenkov VV, Meller J, Kirley TL. Biochemistry 2005;44:8998-9012. [PubMed: 15966724]

16. Chadwick BP, Frischauf AM. Mammalian Genome 1997;8:668-672. [PubMed: 9271669]

17. Mateo J, Kreda S, Henry CE, Harden TK, Boyer JL. Journal of Biological Chemistry 2003;278:39960-39968. [PubMed: 12888562]

18. Vlajkovic SM, Housley GD, Greenwood D, Thorne PR. Molecular Brain Research 1999;73:85-92. [PubMed: 10581401]

19. Wang CJF, Vlajkovic SM, Housley GD, Braun N, Zimmermann H, Robson SC, Sevigny J, Soeller C, Thorne PR. Biochemical Journal 2005;385:729-736. [PubMed: 15362980]

20. Biederbick A, Kosan C, Kunz J, Elsasser HP. Journal of Biological Chemistry 2000;275:19018-24. [PubMed: 10858452]

21. Matsumoto M, Sakurai Y, Kokubo T, Yagi H, Makita K, Matsui T, Titani K, Fujimura Y, Narita N. FEBS Letters 1999;453:335-340. [PubMed: 10405171]

22. Smith TM, Kirley TL. Biochemistry 1999;38:321-328. [PubMed: 9890913]

23. Stoscheck CM. Analytical Biochemistry 1987;160:301-305. [PubMed: 3578757] 
24. Fiske CH, Subbarow Y. Journal of Biological Chemistry 66(1925):375-400.

25. Smith TM, Kirley TL. Biochemistry 1999;38:1509-1516. [PubMed: 9931016]

26. Lennon GG, Auffray C, Polymeropoulos M, Soares MB. Genomics 1996;33:151-152. [PubMed: 8617505]

27. Wang TF, Ou Y, Guidotti G. J Biol Chem 1998;273:24814-24821. [PubMed: 9733785]

28. Grinthal A, Guidotti G. Biochemistry 2002;41:1947-1956. [PubMed: 11827541]

29. Grinthal A, Guidotti G. Biochemistry 2004;43:13849-13858. [PubMed: 15504047]

30. Mukasa T, Lee Y, Knowles AF. Biochemistry 2005;44:11160-11170. [PubMed: 16101300]

31. Knowles AF, Li C. Biochemistry 2006;45:7323-33. [PubMed: 16752921]

32. Kirley TL, Crawford PA, Smith TM. Purinergic Signalling 2006;2:379-389. [PubMed: 17710224]

33. North RA. Physiol Rev 2002;82:1013-1067. [PubMed: 12270951]

34. Bruss M, Barann M, Hayer-Zillgen M, Eucker T, Gothert M, Bonisch H. Naunyn Schmiedebergs Archives of Pharmacology 2000;362:392-401.

35. Feng YH, Li X, Wang L, Zhou L, Gorodeski G. Purinergic Signalling 2006;2:84-85. 


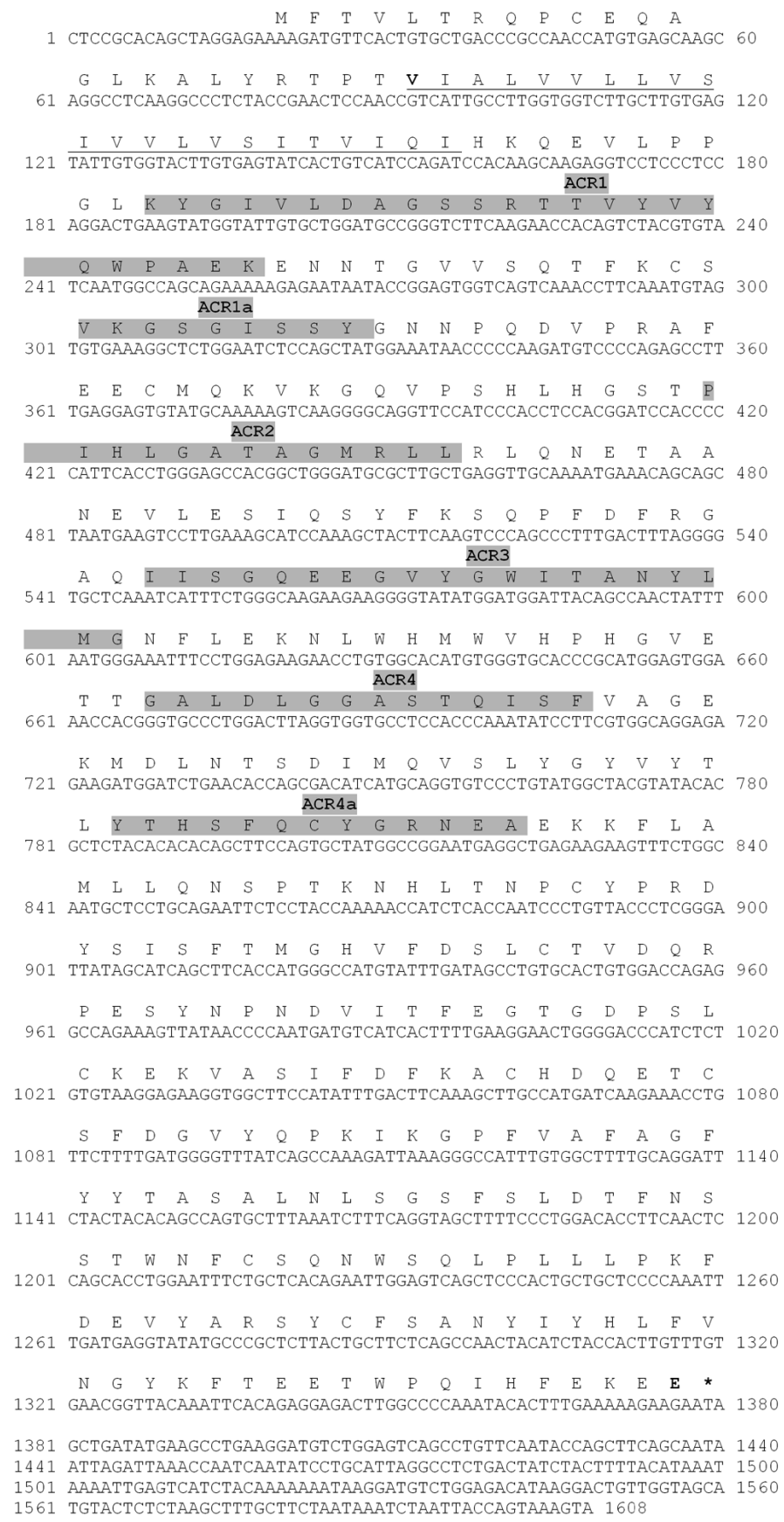

Figure 1. cDNA and translated amino acid sequences of NTPDase3ß, the truncated splice variant of NTPDase3

Amino acids where NTPDase $3 \beta$ differs from NTPDase $3 \alpha$ are indicated in bold. The N-terminal transmembrane domain is underlined. ACR regions are labeled and shaded. 

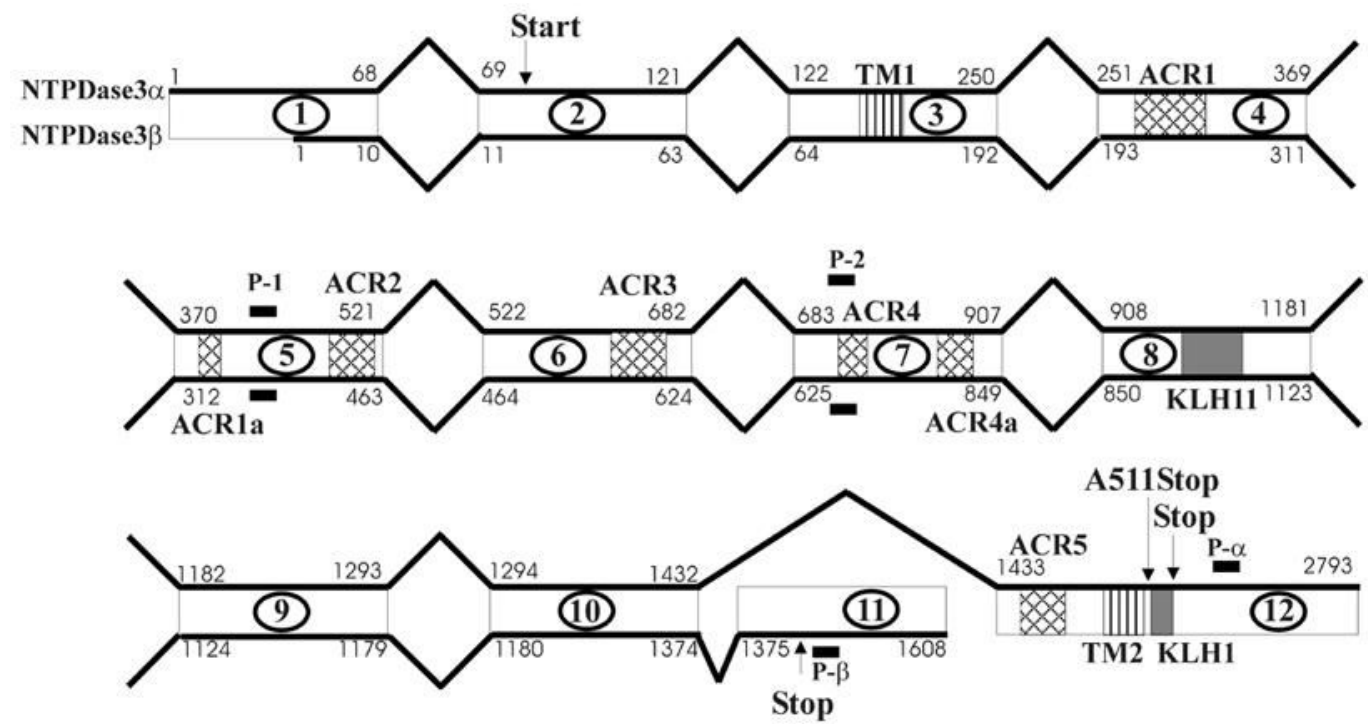

Figure 2. A diagram of the exon map of the human NTPDase3 gene from chromosome 3 representing the splicing events that occur

The boxes represent the 12 exons, which are numbered (exon numbers inside ovals), and the introns are represented by peaks and valleys connecting the exons above and below the boxes. The cDNA sequence is indicated by the thick, dark line with NTPDase $3 \alpha$ above and NTPDase $3 \beta$ below the exons. The seven ACRs are marked by crosshatched boxes within the exons. The transmembrane helixes (TM1 and TM2) are represented by boxes containing vertical lines. The two antisera recognition sequences (KLH1 and KLH11) are indicated by shaded boxes within the exons. The four PCR primers used to selectively identify and amplify NTPDase $3 \alpha$ or NTPDase $3 \beta$ are represented by black bars above the exons for NTPDase $3 \alpha$ and/or below the exons for NTPDase $3 \beta$. 


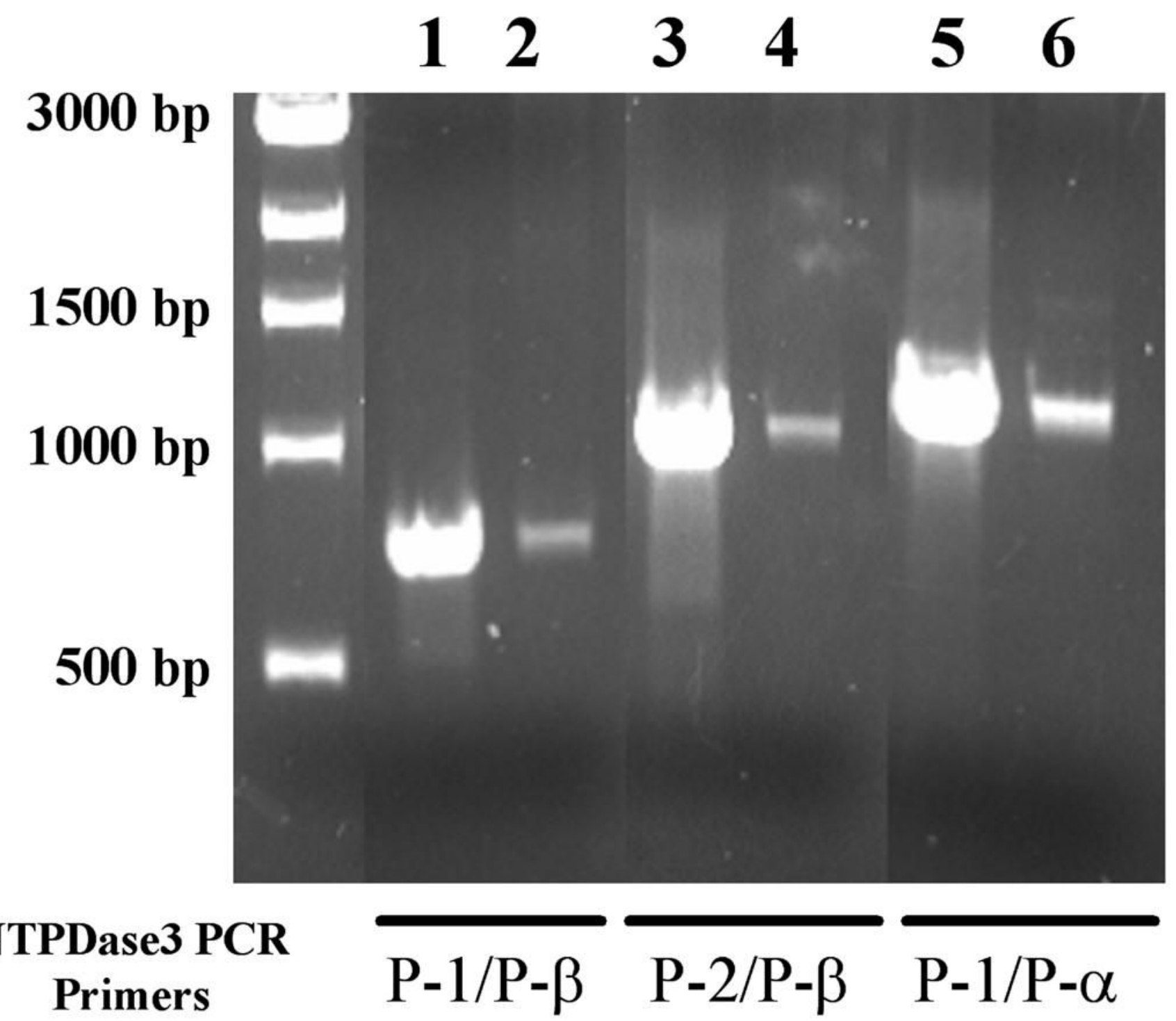

Figure 3. Selective PCR amplification of fragments of NTPDase3ß or NTPDase3 $\alpha$ from a human lung cDNA library

Lanes 1 and 2 use the primers $\mathrm{P}-1$ and $\mathrm{P}-\beta$, while lane 3 and 4 use the primers $\mathrm{P}-2$ and $\mathrm{P}-\beta$ to selectively amplify NTPDase $3 \beta$. Lanes 5 and 6 use the primers P-1 and P- $\alpha$ to selectively amplify NTPDase $3 \alpha$. Lane 2, 4, and 6 contain PCR products from the amplification of fragments of NTPDase 3 cDNAs from a human lung cDNA library using the above primers. Lanes 1, 3 and 5 contain the PCR products from the amplification of fragments from NTPDase $3 \beta$ (lanes 1 and 3) or NTPDase $3 \alpha$ (lane 5) cDNA plasmids. 


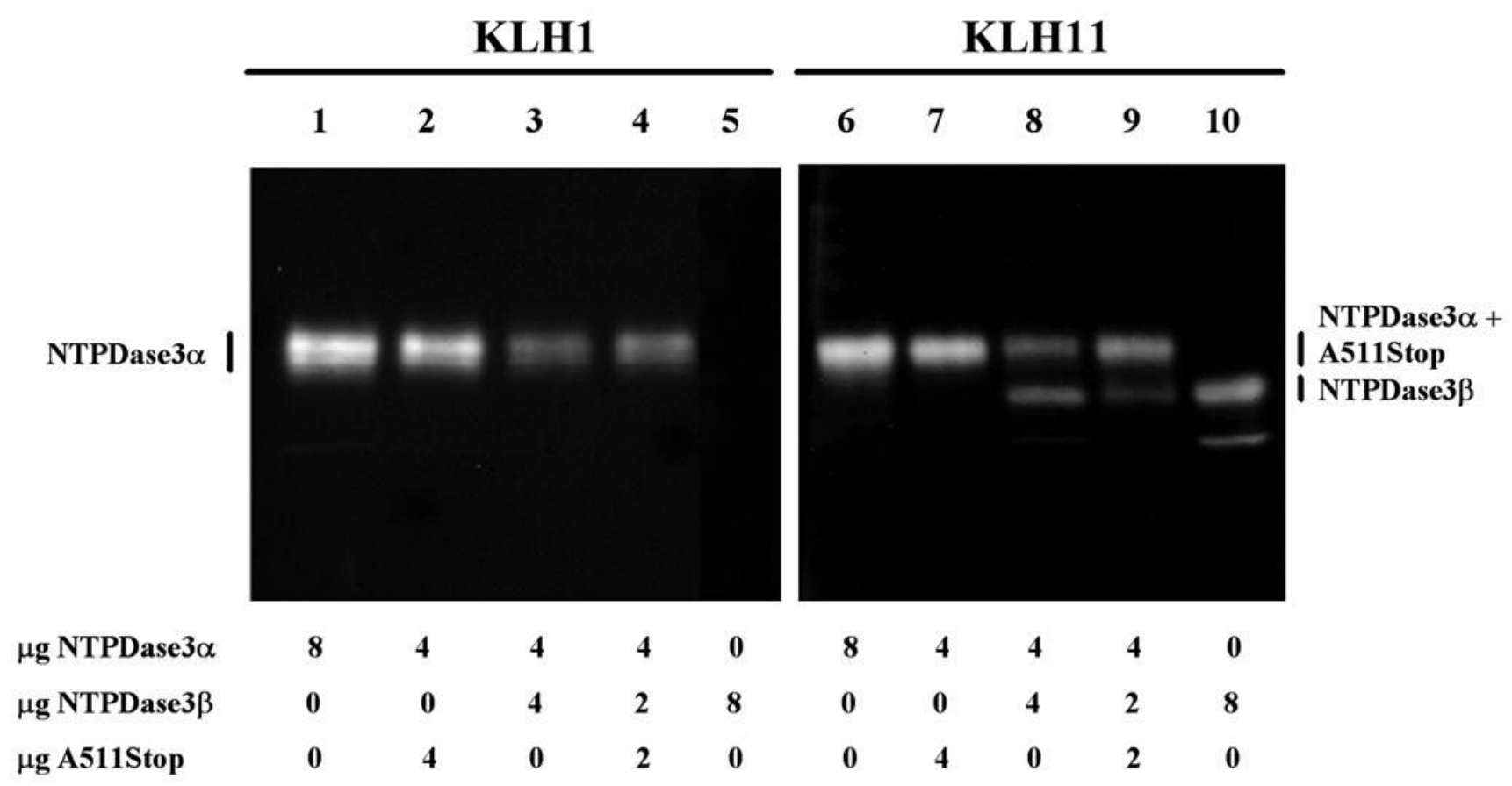

Figure 4. Western blot analysis of co-transfection experiments with KLH1 or KLH11

COS- 1 cells were transfected with a total of $8 \mu \mathrm{g}$ of cDNA as indicated. Primary antibodies used were KLH1 for lanes 1-5 and KLH10 for lanes 6-10. KLH1 is reactive against the Cterminal cytoplasmic amino acid sequence and therefore is only reactive with NPTDase $3 \alpha$. KLH11 is reactive against a portion of the extracellular domain and therefore recognizes NTPDase $3 \alpha$ and A511Stop (active, upper bands) as well as NPTDase $3 \beta$ (inactive, lower bands). 


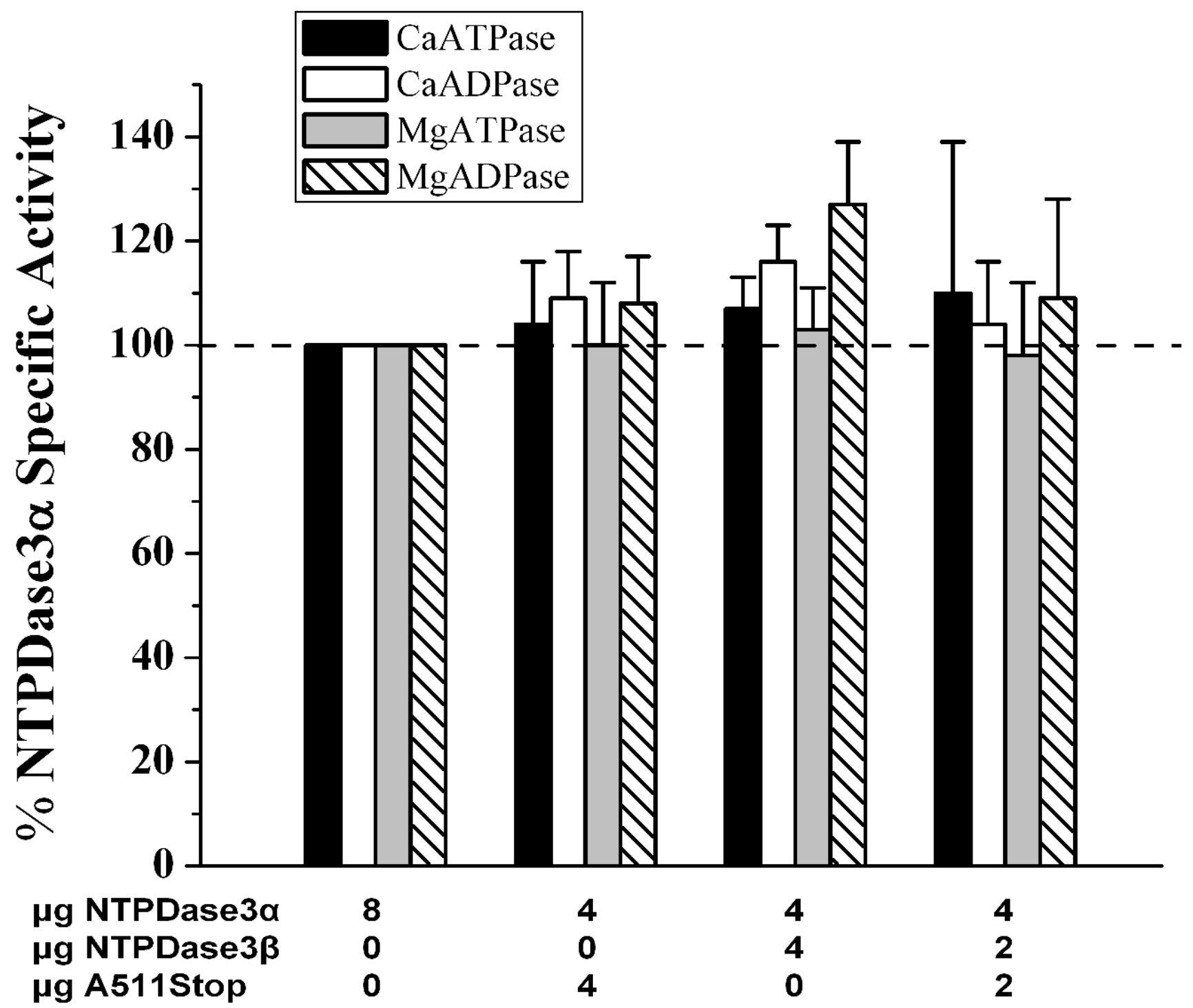

Figure 5. Activity of co-transfections normalized per molecule active protein

Specific activity was calculated from the amount of inorganic phosphate released during the reaction as $\mu \mathrm{mol}$ Pi released per mg active protein per hour (see Table 1) and normalized to the expression level of active forms of the protein, NTPDase $3 \alpha$ and A511Stop, quantified using the upper bands observed in the KLH11 Western blot (see Figure 4, lanes 6-9, and Table 1). This normalized specific activity was used to generate the percentage normalized specific activities, defining the NTPDase $3 \alpha$, KLH11 normalized, specific activity as $100 \%$.

Nucleotidase activities measured were: $\mathrm{Ca}^{2+}$-ATPase, black bars; $\mathrm{Ca}^{2+}-$ ADPase, white bars, $\mathrm{Mg}^{2+}$-ATPase, grey bars; $\mathrm{Mg}^{2+}$-ADPase, hatched bars. Error bars represent the standard deviations for multiple assays performed on three independent transfection experiments. Note that none of the nucleotidase values plotted in this Figure are statistically different from the " $8,0,0$ " control NTPDase $3 \alpha$ values at the $p=0.05$ level of significance. 

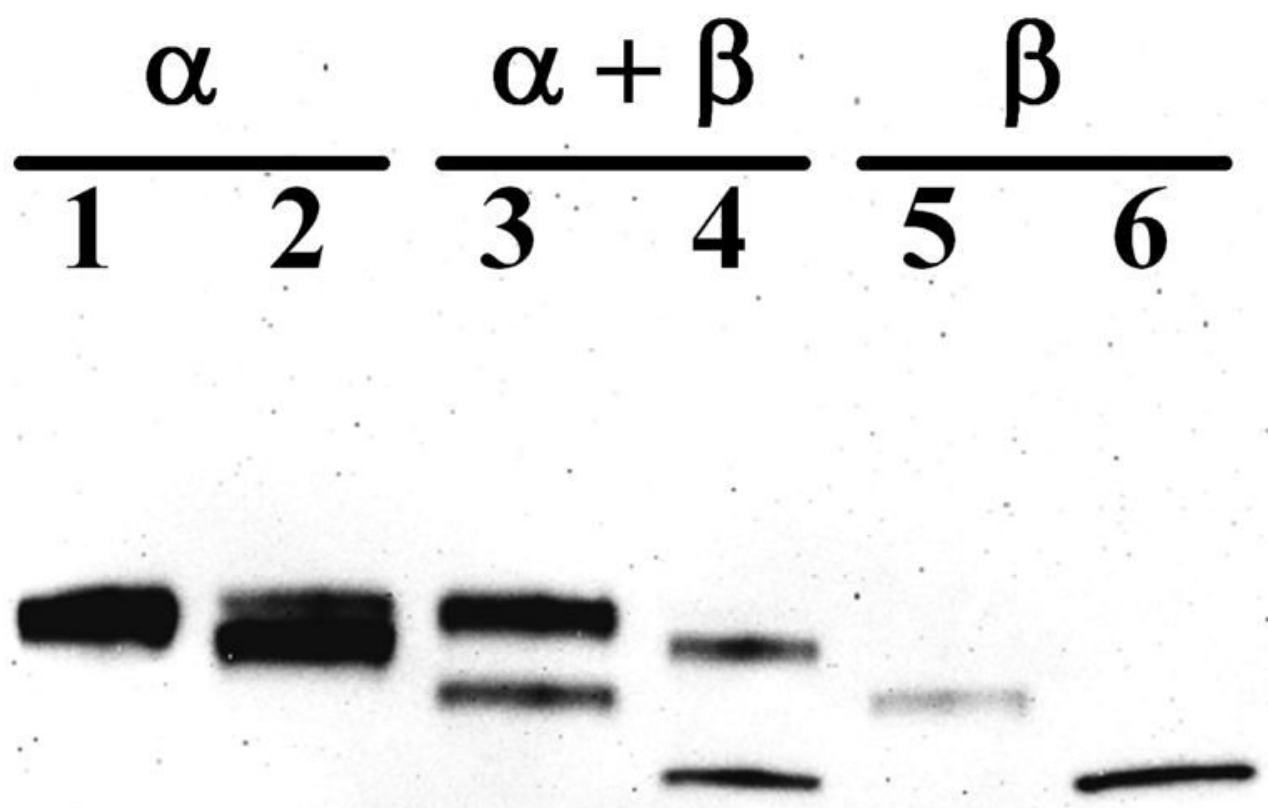

Figure 6. Western blot analysis of COS cell membranes co-transfected with NTPDase3ß and/or NTPDase $3 \alpha$, with and without Endo H glycosidase treatment Lanes 2, 4, and 6 contain Endo H deglycosylated NTPDase3, while lanes 1, 3, and 5 contain control NTPDase 3 samples treated in like fashion, but without Endo H. Lanes 1 and 2 contain NTPDase $3 \alpha$, lanes 3 and 4 contain co-transfected NTPDase $3 \alpha$ and NTPDase $3 \beta$, and lanes 5 and 6 contain NTPDase $3 \beta$. 
Table 1

Results of co-transfections of different NTPDase 3 proteins in COS cells.

\begin{tabular}{|c|c|c|c|c|c|c|c|c|}
\hline \multicolumn{3}{|c|}{$\mu \mathrm{g}$ cDNA used to transfect COS cells } & \multicolumn{2}{|c|}{$\begin{array}{c}\text { Expression levels relative to } \\
\text { NTPDase3a transfected } \\
\text { alone (from Figure 4) }\end{array}$} & \multicolumn{4}{|c|}{$\begin{array}{c}\text { Specific activity }(\mu \mathrm{mol} / \mathrm{mg} / \mathrm{hr} \text {, uncorrected for } \\
\text { expression levels) }\end{array}$} \\
\hline NTPDase3a & 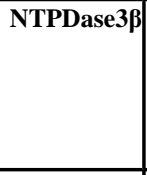 & A511Stop & $\begin{array}{c}\text { Using } \\
\text { KLH1 } \\
\text { detected } \\
\text { bands }\end{array}$ & $\begin{array}{c}\text { Using } \\
\text { KLH11 } \\
\text { detected } \\
\text { active, } \\
\text { upper } \\
\text { bands } \\
\end{array}$ & $\begin{array}{c}\text { Ca- } \\
\text { ATP }\end{array}$ & $\begin{array}{c}\text { Ca- } \\
\text { ADP }\end{array}$ & $\begin{array}{l}\text { Mg- } \\
\text { ATP }\end{array}$ & $\begin{array}{l}\text { Mg- } \\
\text { ADP }\end{array}$ \\
\hline 8 & $\mathbf{0}$ & $\mathbf{0}$ & 100 & 100 & $\begin{array}{r}203 \\
\pm 31 \\
\end{array}$ & $38 \pm 3$ & $\begin{array}{r}119 \\
\pm 29 \\
\end{array}$ & $32 \pm 6$ \\
\hline 4 & $\mathbf{0}$ & 4 & $60 \pm 14^{*}$ & $82 \pm 1^{* *}$ & $\begin{array}{r}173 \\
\pm 20^{+} \\
\end{array}$ & $34 \pm 3^{+}$ & \begin{tabular}{r|r|}
98 \\
$\pm 24^{\#}$ \\
\end{tabular} & $28 \pm 4^{\#}$ \\
\hline 4 & 4 & $\mathbf{0}$ & $30 \pm 6^{*}$ & $34 \pm 5^{* *}$ & $73 \pm 7^{+}$ & $15 \pm 2^{+}$ & $41 \pm 9^{\#}$ & $14 \pm 1^{\#}$ \\
\hline 4 & 2 & 2 & $31 \pm 4$ & $49 \pm 6$ & $93 \pm 4$ & $19 \pm 1$ & $56 \pm 6$ & $17 \pm 1$ \\
\hline
\end{tabular}

Means and standard deviations from 3 independent co-transfection experiments are presented, each assayed in triplicate. The most important comparisons are those between the "4,0,4" row (co-expression of the NTPDase $3 \alpha$ and the active and properly processed A511Stop NTPDase3) and the "4,4,0" row (co-expression of the NTPDase $3 \alpha$ and the truncated NTPDase $3 \beta$ ). The statistical p-values calculated for the differences in the means observed in these two rows are:

* $=0.04$;

*** $=0.003$;

$+=0.004$

${ }^{\#}=0.03$. The means for the nucleotidase activities of the "4,2,2" row (co-expression of NTPDase3 $\alpha$, NTPDase3 $\beta$, and A511Stop NTPDase3) are all also significantly different from the " $4,0,4$ " row, but the p-values are omitted for clarity. The activities are uncorrected for protein expression levels. After correction for the relative expression levels of the upper, active NTPDase3 protein band detected by the KLH11 antibody (depicted in Figure 4 and quantified in this Table), the normalized nucleotidase data is re-plotted in Figure 5 as the percentage of the NTPDase $3 \alpha$ activities (the "8,0,0" row). 